\title{
LA IDENTIDAD CULTURAL COMO ELEMENTO CLAVE PARA PROFUNDIZAR EN LOS PROCESOS EDUCATIVOS QUE APUNTEN A LA CONVIVENCIA EN SOCIEDADES MULTICULTURALES
}

\author{
CULTURAL IDENTITY AS A KEY ELEMENT IN THE PROCESS FOR FURTHER EDUCATION \\ AIMED AT LIVING TOGETHER IN MULTICULTURAL SOCIETIES
}

\author{
Iván Manuel Sánchez Fontalvo*, Wilmer Rafael Aguirre Domínguez** y Juan Carlos Ochoa Triana***
}

\section{RESUMEN}

Es una necesidad imperante que los procesos educativos que se desarrollen en el resguardo indígena Kankuamo apunten a fortalecer la identidad cultural como signo de perpetuar las costumbres, tradiciones y su cosmovisión de pueblo indígena frente a su relación con su entorno, para que la educación propia y los contenidos que exigen los estándares nacionales se articulen formando niños, niñas, adolescentes que amen su territorio y contribuyan a mantener en el tiempo su identidad.

Para fortalecer estos procesos educativos se debe tener como base fundamental la comprensión y visibilizar la manera en que la etnoeducación en la comunidad indígena Kankuama está incidiendo en los procesos de la identidad cultural desde la perspectiva de su cosmovisión y las relaciones interculturales.

Cabe aclarar que los grupos de discusión que se han desarrollado en el marco de esta investigación diagnóstica, han mostrado un acercamiento a los procesos educativos que se llevan a cabo en los establecimientos educativos del resguardo indígena; y pueden ser determinantes en el fortalecimiento de la identidad cultural, de manera que los planes de estudios que están inmersos en los currículos tengan presente la educación propia sin desligar de la educación la perspectiva de la interculturalidad.

Palabras clave: Identidad étnica, cultura, multiculturalidad, interculturalidad y educación.

\begin{abstract}
Is a pressing need for educational processes taking place in the Indian reservation Kankuamo aimed at strengthening cultural identity as a sign of perpetuating the customs, traditions and worldview of indigenous people against their relationship with their environment, so that the own education and contents that require national standards are articulated forming children, teens who love their country and contribute to maintaining its identity over time.
\end{abstract}

Fecha de recepción: Abril 17 de 2015 / Fecha de aceptación: Junio 01 de 2015

Tipología: Artículo de Investigación Científica y Tecnológica

Para citar éste artículo: Sánchez, F. I., Aguirre, D. W., \& 0, T. J. (2015). La identidad cultural como elemento clave para profundizar en los procesos educativos que apunten a la convivencia en sociedades multiculturales. Praxis. Vol. 11, 61 - 75

*Ph.D en Ciencias de la Educación. Docente de tiempo Completo, Universidad del Magdalena. Colombia. Email: ivamas24@hotmail.com

**Magister en Educación Universidad Popular del Cesar. Colombia.Email: wilaguirre08@hotmail.com

***Magister en Educación. Universidad Popular del Cesar. Colombia.Email: juank.ingris@gmail.com 
To strengthen these educational processes must be firmly rooted in the understanding and visualize how ethnic education in the indigenous community Kankuamo is affecting the processes of cultural identity from the perspective of their worldview and intercultural relations.

Clearly note that the discussion groups that have been developed within the framework of this research have shown diagnosed approach to educational processes carried out in educational institutions of indigenous reservation; can be decisive in strengthening cultural identity, so that curricula that are embedded in curricula have this education itself without disentangle education intercultural perspective.

Keywords: Ethnicity, culture, multiculturalism and intercultural education

\section{INTRODUCCIÓN}

$\mathrm{L}$ a esencia de este trabajo investigativo se centró en comprender la identidad cultural como elemento clave de los procesos educativos y visibilizar la manera en que la etnoeducación en la comunidad indígena kankuama está incidiendo en los procesos de la identidad cultural desde la perspectiva de su cosmovisión y las relaciones interculturales.

El método utilizado del grupo de discusión se convirtió en un aspecto relevante al lograr comprender que los procesos educativos pueden fortalecer la identidad cultural, para ello, entendemos a Ferrando (1994) quien afirma que las discusiones de grupo permiten definir la estructura significativa y la dialéctica interna de las posiciones ideológicas latentes o posibles, enmarcadas en la flexibilidad y espontaneidad de los participantes, donde no existe espacio para cohibir los pensamientos y posturas que asuman los integrantes del grupo (p.2).

Además, se puede ampliar esta técnica de recogida de información a través de Krueguer (1991), quien afirma que el grupo de discusión, al basarse en conversaciones cuidadosamente planeadas, diseñadas para obtener información de un área definida de interés, busca lograr constructos comunes originados de la discusión y aportes de todos (p.24).

De esta manera, la metodología planteada sirvió para que los participantes optaran por una actitud abierta en la que se pudo establecer posturas claras y bien diferenciadas del objeto de estudio de esta investigación; además, las respuestas a cada pregunta de la guía socializada fueron ampliamente discutidas con los actores de los grupos de discusión.

Así, se analizarán los datos específicos del objeto de estudio del diseño cualitativo que se referencia en esta tesis: el contexto general de esta investigación, la muestra seleccionada para participar en esta fase de la investigación, el instrumento de recogida de información empleado, así como la previsión del tipo de análisis de la información que se ha desarrollado.

\section{METODOLOGÍA}

La muestra: los actores (docentes y directivos docentes)

En este análisis diagnóstico, la población de referencia son los orientadores de los procesos educativos que se realizan en las diferentes unidades educativas, instituciones y las escuelas anexas del Resguardo Indígena Kankuamo, las cuales están divididas administrativamente así:

- Unidad Educativa de Guatapurí: comprende un colegio y las escuelas de las

- Comunidades de Guatapurí y Chemesquemena

- Unidad Educativa de Atánquez: comprende un colegio y las escuelas de las

- Comunidades de Atánquez, Pontón y Las Flores

- Unidad Educativa de La Mina: comprende un colegio y las escuelas de las 
- Comunidades de La Mina, Los Haticos, Rancho la Goya, Ramalito, El Mojao y Rioseco

Se hizo un muestreo intencional en las instituciones educativas, se abarcó la totalidad de los docentes y directivos docentes de las instituciones seleccionadas: San Isidro Labrador de Atánquez y el Instituto Agrícola La Mina.

En la unidad educativa de Atánquez, el grupo de discusión estuvo conformado por 8 profesoras y 16 profesores, que laboran en los niveles de básica secundaria y media académica de la Institución Educativa San Isidro Labrador. Estos docentes orientan las áreas de ciencias naturales, ciencias sociales, matemáticas, humanidades, informática, religión, educación física y las específicas del saber ancestral.

En la unidad educativa de La Mina, el grupo de discusión fue integrado por 24 docentes, 14 mujeres y 10 hombres, de la Institución Educativa Agrícola de La Mina, ellos se desempeñan en todos los niveles y áreas del conocimiento, incluyendo las asignaturas del saber ancestral que hacen parte de la educación propia.

Para el sociólogo Giddens (1984), la etnografía es el estudio directo de personas o grupos durante un cierto período, utilizando la observación participante o las entrevistas para conocer su comportamiento social para lo que es imprescindible el trabajo de campo como herramienta básica. La investigación etnográfica pretende revelar los significados que sustentan las acciones e interacciones que constituyen la realidad social del grupo estudiado; esto se consigue mediante la participación directa del investigador. Con frecuencia, el investigador asume un papel activo en sus actividades cotidianas, observando lo que ocurre y pidiendo explicaciones e interpretaciones sobre las decisiones, acciones y comportamientos (p.1).

Para Arango (1991), en Colombia los pueblos indígenas han asumido que el derecho a la diferencia está directamente ligado a la capacidad de decisión de cada pueblo sobre el tipo de educación que quiere para sus hijos. Por esa razón, las organizaciones locales, regionales y nacionales han incluido la educación como uno de los componentes fundamentales en sus propuestas de autonomía (p.4). Debido al proceso de colonización y aculturación, los Kankuamos empezaron a olvidar su compromiso de cuidar La Sierra y de mantener sus tradiciones. Según su cosmovisión La Sierra está representada como una mesa de la cual hacen parte los Kogui, Arhuacos, Wiwas y los Kankuamos, y cada uno de ellos son las bases para guardar el equilibrio natural, espiritual y ancestral de La Sierra Nevada de Santa Marta.

Por consiguiente, la finalidad de los grupos de discusión es comprender la identidad cultural como elemento clave de los procesos educativos para aportar a la construcción de convivencia en sociedades multiculturales, y de esta manera poder tener un diagnóstico real y/o contextualizado que permita diseñar un proyecto de investigación educativa que retome las creencias ancestrales de la etnia Kankuama y por ende se brinde una formación y una educación para la ciudadanía intercultural que fortalezca la identidad de este pueblo indígena.

Krueguer (1991, citado en Del Campo), señala que el grupo de discusión puede ser utilizado previamente al inicio de un programa de intervención: puede utilizarse como medio para valorar necesidades o establecer un diagnóstico de una situación determinada.

En el marco de esta investigación el desarrollo de los dos grupos de discusión estuvo compuesto por 24 personas con los siguientes perfiles: profesorado de educación básica primaria, secundaria y media académica, auxiliar administrativo, el coordinador académico y rectores de cada Institución donde se llevó a cabo este trabajo. Se propició un ambiente de libertad y espontaneidad a los informantes para expresar sus propias ideas y opiniones sobre la identidad cultural del pueblo indígena Kankuamo, realidad multicultural y la diversidad, percepciones y actitudes ante estas realidades. El tiempo de duración de los grupos de discusión en cada sesión no superó las 2 horas. Se veló porque el ritmo fuese ágil y la participación equilibrada, teniendo en cuenta que los grupos de discusión estuvieron sujetos 
a las directrices emanadas de la Secretaría de Educación Municipal de Valledupar. Se brindó un refrigerio en cada sesión con el ánimo de favorecer un entorno de discusión confortable.

\section{Iniciación de la dinámica de los grupos de discusión}

Las dos sesiones se iniciaron con una presentación por parte del investigador que coordina el proyecto, quien dio a conocer los aspectos siguientes:

Motivos de la convocatoria: se informó que se deseaba conocer cuál era la percepción del profesorado de las Instituciones Educativas del resguardo Kankuamo frente al ordenamiento del proyecto educativo para la etnia, ante la formación de la ciudadanía intercultural, para desarrollar con fundamentos reales un proyecto de investigación que permita conocer cómo se ha dado la cohesión del PEK frente a las realidades socioculturales de la población indígena base de estudio; además que expresaran sus puntos de vista del proyecto etnoeducativo.

Presentación de los participantes: la presentación se hizo por parte del coordinador, cabe destacar que según las características más importantes del grupo de discusión, específicamente su composición, las personas en lo posible no deben conocerse. Sin embargo, en este trabajo de investigación el profesorado se conoce porque trabaja en la misma institución. Por consiguiente, la presentación básicamente es para que el investigador conozca al profesorado participante. También se recordó el cronograma de los grupos de discusión previamente concertado entre dirección y profesorado y una semana después de haber finalizado la segunda y última sesión, se hará una sesión de devolución de la información más elaborada con sus respectivas conclusiones.

Proporcionar información y concertar el proceso de registro de la información con el profesorado $y$ directivos participantes. En cada una de las dos sesiones de discusión, se dio a conocer que la información recogida es con el ánimo de desarrollar un proyecto de investigación que permita establecer puntos de acuerdos en la educación étnica para fortalecer la identidad cultural de los estudiantes, basado en el conocimiento del profesorado hacía la construcción de la ciudadanía intercultural y que dicha información no será desviada hacia otro aspecto que no sea la investigación y mejora de la educación propia. Lo anterior teniendo en cuenta que el sistema de registro de la información implicó grabar las discusiones en una registradora de audio.

Se inició la dinámica y la formulación de la primera pregunta: se abrió paso a la discusión con la formulación de la primera pregunta escrita en el guion.

\section{Guion de las preguntas que favorecieron la conversación del grupo}

Las preguntas estuvieron cimentadas en la claridad de la formulación para que se diera un buen entendimiento de los interrogantes, se centró en los aspectos principales que aborda esta investigación y se desarrolló ampliamente en el tiempo previsto. De cada uno de los tres temas se desprenden varias preguntas, estas preguntas buscan responder al objetivo general de la investigación diagnóstica. Las preguntas son:

\section{Tema 1: Realidad sociocultural del pueblo indígena Kankuamo y su relación frente al proyecto etnoeducativo Kankuamo}

¿Cuál es la visión que tienen ustedes sobre la realidad sociocultural del pueblo indígena Kankuamo? ¿Qué conocimientos tienen sobre las distintas prácticas educativas aplicadas a los estudiantes de la etnia kankuama y su incidencia en su realidad sociocultural? ¿Cuáles elementos conocen ustedes que sean represantivos de la identidad cultural del pueblo indígena Kankuamo? ¿Las IE están aplicando el proyecto etnoeducativo según la normatividad del Ministerio de Educación Nacional? ¿Por qué?

¿Cómo ven ustedes el sentimiento de pertenencia como colombianos sobre nuestra realidad multicultural? 
Tema 2: La realidad etnoeducativa frente a los procesos identitarios del pueblo Kankuamo

¿Cómo se sienten ustedes cuando en su salón de clases encuentran alumnos de diferentes grupos étnicos y culturas? ¿Cómo perciben ustedes esa realidad? ¿Creen ustedes que el alumnado debe adaptarse a las políticas educativas que ofrece la institución o esta debe responder a la realidad multicultural del alumnado? ¿Creen ustedes que las diferencias étnicas y culturales inciden en la comunicación positiva en la institución? ¿Qué hacen ustedes cuando se presenta un caso de agresión estereotipada entre alumnos con diferencias étnicas y culturales? ¿Qué actitud debe asumir el profesorado ante la lluvia de estereotipos y prejuicios difundidos en el contexto escolar?

Tema 3: El proyecto etnoeducativo kankuamo (PEK) frente al fortalecimiento de su identidad cultural

¿Se promueven en la escuela aspectos culturales y personales del otro, sobre la base del respeto a la diversidad? En este sentido, ¿Se esta promoviendo la preservación de la identidad cultural mediante la participación del alumnado en eventos culturales autóctonos? ¿Se respeta y estimula el derecho a las expresiones culturales, lingüísticas de las personas de los grupos étnicos? ¿A través de la educación en este centro educativo están o no desarrollando una conciencia y valoración de la diversidad étnica y cultural? ¿Tienen alguna experiencia que comentar en este campo? ¿Cómo valoran la experiencia? ¿Existen dinámicas inconscientes en la institución que tienden a la base: prejuicios, estereotipos que llevan a la discriminación? ¿Qué símbolos (personas, edificios, banderas, himnos, elementos de trabajo, utensilios caseros, instrumentos musicales, danzas, etc.) ayudan a la cohesión social de la etnia? ¿Han propiciado en el centro educativo espacios para que el alumnado cree sus propios símbolos? ¿Con qué fundamentos formularon el plan de área y de clase para el 2013? ¿Creen que su contenido forma para la participación ciudadana intercultural? ¿Su propuesta educativa tiene como base la información y formación en los deberes y derechos humanos y las relaciones interculturales? ¿Los alumnos toman parte activa en las decisiones que atañen a la institución educativa para fortalecer su identidad cultural? ¿La organización escolar promueve la vida democrática respetando sus costumbres y creencias? ¿Qué actos y acontecimientos diarios lo demuestran?

\section{RESULTADOS}

Sistema de categorías surgidas durante y después de los grupos de discusión fundamentados en la teoría

\section{Categorías y sub-categorías}

- La identidad y su realidad sociocultural

- Conocimiento ancestral y sitios sagrados

- Iconos representativos del pueblo Kankuamo

- Eventos culturales autóctonos

- La identidad cultural y sus procesos educativos

- El proyecto etnoeducativo Kankuamo (PEK)

- La diversidad de grupos étnicos y culturas en el aula

- Proyectos transversales

- La realidad multicultural

- Diversidad humana cultural

- Educación para la diversidad

Estudio diagnóstico encaminado a tomar la identidad cultural como elemento clave para profundizar en los procesos educativos que apunten a la convivencia en sociedades multiculturales.

Los objetivos específicos que persiguen el fortalecimiento de la identidad cultural a través de los procesos educativos son:

- Analizar la percepción, actitud, comprensión y valoración que se tiene de los procesos educativos frente al fortalecimiento de la identidad cultural

- Detectar los factores que hacen que los procesos educativos en la comunidad indígena repercutan en su realidad socio-cultural

- Develar el sentido que atribuye la comunidad indígena kankuama a la etnoeducación teniendo en cuenta los puntos de encuentros y tensión planteados por la educación propia, occidental y la identidad 
Tabla 1

Aportes de los integrantes de los grupos de discusión

\begin{tabular}{|c|c|c|c|}
\hline TEMAS DE GRUPO & $\begin{array}{c}\text { GRUPO DE } \\
\text { DISCUSIÓN \#1 }\end{array}$ & $\begin{array}{c}\text { GRUPO DE } \\
\text { DISCUSIÓN \#2 }\end{array}$ & TOTAL \\
\hline $\begin{array}{c}\text { Conocimiento } \\
\text { ancestral y espiritual }\end{array}$ & 8 & 2 & 10 \\
\hline $\begin{array}{c}\text { Iconos } \\
\text { representativos y } \\
\text { actos culturales }\end{array}$ & 7 & 3 & 10 \\
\hline $\begin{array}{c}\text { Proyecto } \\
\text { Etnoeducativo } \\
\text { Kankuamo }\end{array}$ & 5 & 2 & 13 \\
\hline $\begin{array}{c}\text { Diversidad de grupos } \\
\text { étnicos y culturas }\end{array}$ & 5 & 3 & 7 \\
\hline $\begin{array}{c}\text { Educación para la } \\
\text { diversidad }\end{array}$ & 5 & 2 & 7 \\
\hline $\begin{array}{c}\text { Proyectos } \\
\text { transversales }\end{array}$ & 4 & 3 & 7 \\
\hline $\begin{array}{c}\text { Diversidad humana } \\
\text { cultural }\end{array}$ & 5 & 4 & 9 \\
\hline $\begin{array}{c}\text { Realidad } \\
\text { sociocultural y PEK }\end{array}$ & 5 & 31 & 78 \\
\hline Identidad cultural & 47 & 4 & 7 \\
\hline TOTALES & 5 & 2 & 76 \\
\hline
\end{tabular}

Fuente: Elaboración propia resultante de la aplicación de la guía a los grupos de discusión.

En virtud al trabajo de campo realizado, se proyectan la reflexión y conclusiones respectivas, teniendo en cuenta una perspectiva hermenéutica de lo obtenido en los dos grupos de discusión.

\section{Conocimientos ancestrales y espirituales}

La pérdida de la identidad cultural a través del tiempo se ha hecho ineludible debido a la influencia de la cultura occidental dentro del resguardo, lo cual trae consigo el hecho de que en la actualidad algunos Kankuamos desconozcan en gran medida sus propias raíces, y es la preocupación de gran parte de este pueblo, sobretodo de la población adulta y no es para menos, los adultos mayores que son quienes poseen esos conocimientos ancestrales, algunos han muerto, otros hoy día no tienen lucidez, solo un pequeño porcentaje de ellos pueden compartir sus conocimientos por medio de la tradición oral, sirviendo esto como base fundamental en el proceso de rescate de la cultura propia.

"La lengua es que cada cosa se nombre por su propio nombre de pronto no decir allá viene Aquileo no allá viene el señor Rector la lengua para nombrar a cada uno con el nombre que le corresponde y en el caso nuestro el rescate, la apropiación de esos elementos lingüísticos que están en el territorio y que 
nosotros los usamos diariamente de una manera de pronto inconsciente comenzar a nombrar con su propia lengua de nosotros y la cultura nuestra tradición que tiene un espacio propio de formación los que desean conocer nuestra identidad, nuestra educación propia bueno hay unas Kankuruas unas lomas unos sitios sagrados donde uno puede ir pero el que no va allá debe hacer eso que ustedes están haciendo escribirlas"GD (La Mina).

La lengua es uno de los factores fundamentales de cualquier cultura, y es por ello que se hace de vital importancia el rescate de la misma; no obstante esto constituye un trabajo bastante arduo porque no simplemente debe hacerse el rescate de los vocablos propios sino que además se debe comenzar a impartir este conocimiento dentro de las instituciones teniendo en cuenta la multiculturalidad y que a pesar de que en su gran mayoría sean Kankuamos no todos están dispuestos a asumir este aprendizaje y adoptarlo como forma de vida.

"Bueno en el modelo educativo también nosotros es de la enseñamos a través de la pedagogía del consejo y del ejemplo yo pienso que un ejercicio de ese ejemplo son las decisiones que se toman comunitariamente, para tomar una decisión dentro de la institución se cita a la comunidad $y$ en consenso se toman las decisiones de lo que finalmente se va a realizar y yo creo que es un ejemplo que se esta dando en los mismos niños, sobre las problemáticas o dificultades que se manejan dentro del aula de clases también hay que irlos haciendo en esa misma dirección tomándolas en conjunto y que los niños tengan la oportunidad de decidir y opinar lo que ellos están pensando" GD (La Mina).

Dentro de este proceso de rescate de la cultura propia, el profesorado hace parte fundamental, debido a que de éstos depende su éxito o fracaso. Cabe anotar que es importante que se estimule este rescate dentro de la institución porque son ellos los integrantes de la sociedad futura y por esta razón la escuela es el lugar propicio puesto que no solamente se formaran personas con conocimientos de las distintas áreas del conocimiento que le servirá para su futuro, sino que ofrece una formación integral que incluye conocer, aprender, y adoptar como forma de vida la cultura propia.

Es importante para las instituciones del Resguardo rescatar su cultura dando a conocer a sus niños cuáles son sus orígenes, que estos aprendan a querer lo que son, a identificarse por su propia cultura y no por una cultura que invadió la propia y que hoy ha obligado a sus habitantes a rescatar para que no desaparezca; para toda cultura se hace indispensable mantener esas costumbres y tradiciones que son las que los identifican y diferencian de las demás, y mostrar también a las demás culturas quiénes son los Kankuamos, que vean quiénes son en realidad es "mantenerse viva su esencia, no perder su identidad”.

"Bueno lo espiritual solo se puede entender desde lo espiritual, si vamos a entender lo espiritual desde lo material entonces muchas cosas no tendrían sentido entonces hay unas normas, por ejemplo yo tengo mi poporo eso me permite a mi poder tener acceso a la Kankuruas de alto nivel en la sierra, pero sin ese elemento no puedo llegar allá entonces para poder hacer eso se necesita una autorización espiritual para poder hacerlo, si lo hago sin autorización entonces estaría robando, estaría siendo ilegal y eso podría dañarme a mí y dañar el equilibrio de la naturaleza por eso para ir a ese sitio hay que cumplir unos pasos para poder llegar allá y eso funciona así para proteger el conocimiento" GD (La Mina).

Para la cultura Kankuama cada característica inmersa en ella posee un significado profundo, ancestral e invaluable; razón por la cual la cuidan tan celosamente, pero también desean rescatar y promover sus prácticas entre sus miembros, esta cultura está basada en lo espiritual y un gran respeto hacia esta clase de cosas. La escuela en su esencia es un ente formador, razón por la cual se ha querido promover la cultura propia dentro de este contexto, que es por supuesto el más propicio para ir formando los miembros futuros de esta cultura, no solo con el conocimiento de la cultura propia, sino que se apropien de este conocimiento y lo transformen como estilo de vida lo cual generaría el tan anhelado rescate de su cultura. 
"La visión sociocultural del pueblo indígena Kankuamo dentro del plan de salvaguarda esta la búsqueda de la recuperación de identidad y de los símbolos que representan a la comunidad, el pueblo indígena se visualiza en unos años como un pueblo reconocido no solamente a nivel nacional sino también por los organismos internacionales como un pueblo autónomo con su gobierno propio, sus instituciones propias y con autonomía independiente al gobierno occidental" GD (La Mina).

El pueblo Kankuamo tiene claras sus intenciones, no solamente de rescatar su cultura sino que además su intención es convertirse en un pueblo con un gobierno propio, un pueblo autónomo e independiente del gobierno nacional, donde las únicas leyes y creencias sean las propias, lo cual quiere decir que su rescate es total y también su anhelo de liberación de la cultura occidental, cuya influencia ha llevado al detrimento de la cultura propia y es tal vez esa la razón por la cual esta cultura desea un gobierno propio para de este modo resguardar su cultura.

\section{Proyecto Etnoeducativo Kankuamo}

El pueblo Kankuamo, en su preocupación por el rescate y el fortalecimiento de la cultura propia desarrolló un nuevo proyecto educativo que propendiera por el fortalecimiento de su cultura mediante la educación, es una forma de recuperar y fortalecer su cultura mediante la etnoeducación, proceso en el cual al estudiante no solamente se le imparte la educación normal, sino que además se imparte el conocimiento de su propia cultura, se crean espacios donde el estudiante escucha y también participa, crea y aprende de sus raíces.

Este proyecto contiene en su esencia esa preocupación por parte de las autoridades y muchos de sus habitantes de permanecer a través del tiempo como pueblo y como cultura. Teniendo en cuenta que hoy en día dentro de este pueblo no solamente conviven Kankuamos, hay cruces de razas, muchas personas que hoy son adultas desde que nacieron vivieron con características de otra cultura diferente a la propia, esto hace de este proceso algo complejo pero no imposible y es por esto que el fortalecimiento se esta impartiendo desde la escuela, porque son ellos la sociedad futura y si se inculca en ellos el valor y el amor por lo propio, éstos lo brindarán con el mismo amor, valor y conocimiento a sus hijos, a los hijos de sus hijos, y de ese modo se dará generación tras generación, el proceso es arduo y debe ser continuo, lleno de amor y motivación para que pueda ser recibido de la misma forma. Es menos complejo fortalecer desde este punto la cultura en personas que a parte de ser niños y adolescentes se hallan en una etapa precisa para ser moldeados.

"Nosotros partimos de un principio de lo que es el modelo educativo, y es que la educación sirvió para aculturizarnos para utilizar ese término, y con el mismo proceso educativo lo que queremos es el rescate de la cultura propia, desde muchos espacios propios; pero nuestra realidad actual es que como resultado de la conquista y de la intromisión de afuera hoy día tenemos un pueblo que es muy diferente a los pueblos tradicionales, porque cuando ustedes llegan aqui ustedes ven por ejemplo la formación propia no se llega a estos espacios y es algo que ya llego y que tenemos que convivir con eso, las mismas creencias religiosas, los evangélicos, los católicos esa es una realidad que ya tenemos, la música donde de pronto ya vamos a escucha un reggaetón, pero también hay unos espacios donde nos sentamos, conversamos con los Mamos, poporeamos en Kankuruas de paja donde ellos también nos dan su mensaje alrededor de un fogón, pero tenemos una realidad sociocultural donde con nuestra gente que pretendemos, que lo que hay no es sacarlo sino progresivamente evitar que cosas que siguen entrando nos sigan deteriorando como las religiones, eso sí le hizo daño a las comunidades indigenas y todavía hoy en día siguen en esa misma orientación, las misma políticas neoliberales, la explotación de la madre tierra, hay gente que le apunta a eso, y tenemos que propender desde la misma cosmovisión a proteger lo propio lo sagrado" GD (La Mina).

El pueblo reconoce como una realidad muy palpable el hecho de que la cultura kankuama ha sido influenciada por la cultura occidental, y que si bien es cierto que no se puede cambiar esto en 
un instante, ni tampoco expulsar a las personas que adopten esta cultura, lo que sí se puede es ir rescatando espacios para las personas que estén interesadas con el animo de que este espacio vaya contando cada día con más personas y que sean estas personas quienes vayan concientizándose de la importancia de lo propio de la esencia del ser y no del imitar o adoptar culturas ajenas. A medida que este proceso se vaya dando y que las personas observen la importancia de lo que es defender las raíces, y de las actividades que estos realizan, entonces se irán vinculando más y más personas a este proceso de rescate y fortalecimiento cultural.

La influencia de la cultura occidental ha traído consigo indudablemente el detrimento de la cultura kankuama en cuanto al vestir, la música, comida, costumbres, religión; todo esto se ve reflejado en sus habitantes y en el cambio en sus estilos de vida, razón por la cual el pueblo mismo se dio a la tarea de resignarse a ver cómo día a día desaparece lo que son. El proceso obviamente es complejo, arduo y necesita además de mucha paciencia y dedicación, y puede decirse además que ha sido acertado que esta necesidad de rescate y fortalecimiento se haya implementado desde la escuela, que haga parte de la formación de cada niño, de cada joven, porque en sus manos estará este pueblo mañana y se tiene todo el tiempo que dura el proceso de formación escolar para lograr no solamente formar estudiantes con conocimientos que le servirán en estudios posteriores tendientes a una mejor calidad de vida, sino en la formación integral de un Kankuamo que sabe quién es, de dónde viene, y que tiene sentido de pertenencia y ama lo que es. Dando como resultado esta mezcla un Kankuamo integral que no solo recibió una formación escolar con miras a un futuro mejor para sí mismo sino alguien que va a formarse para defender y luchar por su pueblo.

La formación religiosa es otro tema al que es difícil acceder y formar porque, independientemente de la cultura a la que pertenezcamos, siendo colombianos todos tenemos derecho a la libertad de culto y por lo tanto en ese tema, a pesar de querer rescatar las creencias como parte viva de la cultura, se debe trasmitir como un conocimiento que según el criterio y creencias de cada niño y joven irán haciendo parte de la vida y de las creencias de estos. No obstante, hoy ya existen varios tipos de religiones inmersas dentro de este resguardo indígena y que no pueden ser expulsados, y que estas personas sean obligadas a cambiar en un instante lo que para ellos constituye su razón de ser; pero sí se le puede mostrar desde la escuela al estudiante para que este conozca una parte importante de su cultura y que sea él mismo quien decida si adoptar o no esta doctrina como propia, de igual forma quienes no lo acepten como propia al menos si sabrán de donde vienen y el significado de muchas cosas que hacen parte de sus raíces.

"A ver, el modelo educativo plantea la existencia de nosotros ahora y mañana y nosotros consideramos como pueblo que habitamos la Sierra Nevada que debemos vivir en convivencia o sea debemos saber vivir y consideramos a los demás pueblos del mundo como hermanos y a los que se creen que no son indigenas que no tienen sus raíces indigenas también los consideramos hermanos y a partir de alli la formación de nuestros estudiantes va orientada a podernos conocer y a poder convivir en este mundo" GD (La Mina).

El modelo educativo considera a su pueblo como habitante indígena de La Sierra Nevada de Santa Marta, que está llamado además a la convivencia pacifica con el resto de las personas sin distingo alguno, sin importar su origen, este tipo de educación encamina a sus estudiantes a la sana convivencia, a la tolerancia, al amor por el prójimo porque es el hermano sin importar dónde haya nacido, a conocerse y a saber que, aunque se sea diferente muchas veces en creencias y cultura, se debe respetar y querer como hijos del mismo mundo.

La etnoeducación como formación, en la que el estudiante es educado de manera integral, ya que este proceso no contiene solamente en su esencia el fortalecer procesos educativos, sino que fortalece los derechos propios, lo cual hace parte de este rescate como pueblo cultural; es importante además que dentro de la formación educativa se le den a conocer sus derechos como indígenas, 
que sepan que además de tener derechos como ciudadanos comunes también hay leyes que los rigen y los protegen como parte de un pueblo indígena, brindando de este modo al estudiante una formación en cuanto a sus derechos constitucionales y también los derechos ancestrales, que estos derechos no han sido dados por parte del Estado como un regalo por ser indígenas, sino por la lucha de muchos indígenas por su derechos, por mostrar que son la raíz de todas las culturas y razas existente en este país y que por ello merece respeto y ser tenido en cuenta por lo que son y lo que valen.

"Cuando hablamos de estudiantes hay una concesión que inicia desde que uno nace hasta que uno muere hay personas que estamos siguiendo un proceso de formación tradicional desde los espacios propios que no necesariamente son estos espacios así cuadriculados, la información que tengo yo es que se está llevando esa parte del fortalecimiento desde la cosmovisión propia, el conocimiento de la lengua, el territorio son como unas asignaturas propias, el territorio, la cosmovisión, la tradición y si se está llevando esa formación pero más que todo en gente ya adulta en gente mayor y en el conocimiento que es el poporo, que significa la mochila, porque tenemos que llegar descalzo a un sitio ese es como el modelo educativo invisible, que lo estamos nosotros llevando, entonces eso se está trabajando, pero paralelo a eso también hay en esa realidad que tenemos otros intereses por las mismas costumbres que fueron impuestas y a veces eso perturba el normal desarrollo de nuestro proceso educativo porque más que todo son tendencias religiosas eso ha afectado bastante de hecho la cosmovisión que hemos planteado nosotros pues no ha sido bien aceptada porque se cree que es una religión y la cosmovisión no es una religión nunca va a ser una religión entonces nosotros ahi hemos tenido como un choque con algunas personas pero más que todo por las tendencias religiosas" GD (La Mina).

El fortalecimiento cultural en lo referente a la cosmovisión ha sido afectado por las religiones, muchos no lo aceptan porque ven en ella una religión distinta a la suya, lo cual coarta el hecho de que se imparta este conocimiento a muchas de estas personas, esta educación contiene lo que es la lengua, el conocimiento acerca del territorio, la tradición oral, conocer el significado de algunas cosas que los identifican como lo son el poporo, la mochila, el porqué se debe entrar a los sitios sagrados descalzos, por qué no se debe hacer ruido, por qué se deben cumplir ciertas normas en estos lugares, y por qué quien incumpla dichas reglas debe pagar un castigo, por qué a esos lugares se debe ir con alguien que tenga el conocimiento o con un mamo. Esta figura en sí constituye para muchos religiosos o cristianos la figura de otra religión que usa otra clase de métodos de creencias y no es cierto, estas personas son conocedoras de la cultura, realizan trabajos tradicionales que en esencia consisten en desear algo, en sostener un hilo un instante, y que sea el universo y la madre naturaleza quienes juntas actúen para proporcionar su deseo, entonces es aquí donde entran a diferir cosmovisión y cristianismo.

"La educación nos brinda la oportunidad de enseñarles a nuestros estudiantes, cosas culturales de nuestra identidad que ya se han perdido, y por otro lado el modelo educativo como lo es el proyecto (PEK) tenemos esa posibilidad de fortalecer la identidad que están comentando" GD (Atánquez).

El diseño de este modelo educativo ha permitido un fortalecimiento cultural dentro de la cultura kankuama, que dentro del aula no solo se enseñe matemáticas, sociales y demás, sino que se imparta conocimiento de la cultura propia que se ha perdido con el pasar de los años. Este contexto favorece notablemente el desarrollo de muchos métodos y estrategias para llegar de forma segura y eficiente al estudiante; que se cree la suficiente motivación, para que de este modo se logre el objetivo, que es el fortalecimiento de la cultura propia, sin olvidar que este proceso es progresivo $\mathrm{y}$ a veces puede darse de forma lenta.

"Dentro del modelo educativo, la educación tiende a ser más investigativa de que el muchacho se apropie del conocimiento, del contexto social donde vive e investigue las razones de cómo estamos formado como sociedad, también hago diferencia, en el sentido que la educación 
tradicional solamente nos muestra un sendero mientras que este muchacho de aqui lo esta viviendo en contacto directo, él asimila con sus cincos sentidos qué es lo que está sucediendo y ahi es donde apunta nuestro modelo educativo a que nuestros estudiantes sean investigativos, que aprenda a valorar su cultura" GD (La Mina).

El modelo educativo está llamado a formar estudiantes con espíritu investigativo, que aprendan a través de lo que ven y de las vivencias propias, que no solamente se formen en lo académico, sino en lo cultural y que aprendan las cosas que no saben de su cultura a través de la investigación con sus padres, familiares, o los mayores conocedores de esos temas, lo cual será para estos niños y jóvenes una experiencia mucho más enriquecedora, puesto que ellos, por sus propios medios buscarán la forma de saberlo y escuchar de labios de quienes han vivido o escuchado de esos temas que ellos pretenden indagar, dando paso de este modo a un aspecto muy importante dentro de esta cultura que es la tradición oral.

"El modelo busca fortalecer el legado cultural, entre ello, las danzas, la parte artesanal, la oralidad, la parte investigativa, es rescatar todo lo que se ha perdido. Dentro de la institución la intensidad horaria es más amplia porque ha habido nuevas asignatura que apuntan a los saberes propios buscando fortalecer la parte cultural. Dentro del PEI de la educación occidental como le llamamos acá, se manejan las asignaturas que nos exige la ley general de la educación. Pero acá tenemos otras asignaturas del saber propio y se amplia la intensidad horaria y la carga para otros docentes" GD (La Mina).

Es interesante este modelo educativo, pues de cierta forma se halla compuesto por una fusión entre educación occidental como ellos le llaman y la educación propia, razón por la cual se ve ampliado el horario de clases para los estudiantes y la necesidad de otros docentes formados y con conocimientos en estas áreas; pues la cultura kankuama es muy amplia y pretende dar a conocer y enseñar a sus estudiantes todo lo referente a la cultura propia con toda la intención de rescatar y fortalecer su cultura, lo cual se constituye de vital importancia dentro del proceso de formación de los estudiantes, porque no solo se les forma en lo académico, sino que además se le a da conocer sus raíces, a saber muchas cosas que son importantes y que a pesar de que él hace parte de esta cultura por su edad, o por falta de esa trasmisión de la tradición oral no conoce.

"Una de las cosas que tiene el modelo... la pedagogía del ejemplo... son metodologías... la investigación directa de ir a ver de primera mano las cosas y la pedagogía del consejo y el ejemplo que hay muchos conocimientos de parte de los mayores de gente que sabe y que los estudiantes no. El fortalecimiento de los trabajos que se hacen en la comunidad que sean colectivos, que todos participen eso permite que el alumno sea responsable y solidario que miren por el bien ajeno. La recuperación de la tradición oral y está articulado con el plan clase... tanto apropiarse del conocimiento, como para que siga la tradición" GD (La Mina).

La investigación es uno de los ejes de este modelo educativo, pues la intención de esta metodología es buscar que el estudiante por sus propios medios conozca de su cultura, haciendo de este un método menos formal y más eficiente para que el estudiante aprenda, además el consejo y el ejemplo hacen parte de este modelo educativo. El estudiante debe escuchar y también tiene todo el derecho a expresarse y serán los consejos y los ejemplos los que encausarán el conocimiento, los ejemplos son métodos más simples pero contundentes en el aprendizaje.

Además, se promueve el trabajo en comunidad, donde se brinde la oportunidad a todos y cada uno de sus miembros con el fin de que el estudiante aprenda a ser colaborador, que se preocupe por ayudar a otros y no de velar únicamente por el interés individual.

Este modelo educativo es como un gran engranaje donde cada cosa va unida a la otra y si una falla todo el proceso se afecta, por esta razón es importante que cada uno de estos aspectos actúen de modo conjunto y que uno fortalezca el otro para que se puedan obtener buenos resultados en el proceso. 


\section{Educación para la diversidad}

"Hay una frase que utilizó José Martí, dice que no hay igualdad social posible sin la igualdad cultural, al darse a conocer el pueblo Indígena Kankuamo en la parte de occidente da a conocerse ante las demás comunidades y dentro de las demás poblaciones y así mismo el pueblo indígena Kankuamo debe conocerlo, el mundo externo, y esa igualdad permite mejores condiciones para el pueblo" GD (La Mina).

La igualdad entendida desde la óptica del pueblo indígena se refiere a una igualdad total; no solo como parte de una sociedad, sino como parte de una cultura que no sea excluida, sino tenida en cuenta por lo que son indígenas, que dicha igualdad les sirva como base para darse a conocer en cada rincón posible, y que además las condiciones de su pueblo mejoren sin desconocer sus costumbres.

"Yo no he tenido oportunidad de trabajar con otro grupo, otros estudiantes de otro grupo étnico, pero yo tuve la oportunidad de hablar con la profesora Ludís la profesora que ella trabajaba en Murillo, en la comunidad de Murillo me explicaba que ella tenía allá estudiantes Arhuacos y yo pienso, o sea como me contaba ella y a mi también me parece la experiencia que debe ser muy agradable el compartir con ellos su lengua todas sus experiencias de ellos, que uno a través de esas experiencias uno adquiere más conocimientos" GD (La Mina).

La educación desarrollada en el contexto de la multiculturalidad es una experiencia muy enriquecedora para todos los que participan de ella (docentes, estudiantes); puesto que dentro de este proceso de formación hay varios elementos distintos en la educación. Primero, porque dentro del proceso de formación está contenida no solamente la formación académica, sino que además se enseña a los estudiantes de su cultura impulsando de este modo el rescate por la cultura propia, y además de esto con la presencia de personas de otras etnias se hace presente un aprendizaje a través de la oralidad sobre otras culturas, y se da un proceso interesante más que todo para el docente, porque dentro de este proceso no solamente es un formador sino también un aprendiz, a través de las experiencias, del compartir, del observar cómo interactúan varias culturas dentro del contexto de la educación.

"Bueno ahorita mismo nosotros contamos con la construcción del modelo y encontramos personas mayores, inclusive profesores que ya se visten de blanco, yo pienso que como primero los estudiantes no veían a los estudiantes de los otros pueblos hermanos con mantas y cuando los veían con mantas les causaba gracia, yo trabaje en el colegio de Rio Seco un ciclo y allá iba un Arhuaco con su manta y normalmente los compañeros jugaban con él y no había problema, los estudiantes ya se han concientizado del uso del vestido ya por ese lado no va a haber problemas" GD (La Mina).

Para el pueblo indígena Kankuamo tiene importancia recuperar cada uno de los elementos que hacen parte de su cultura. Por ejemplo su vestuario, puesto que para ellos es una de las características más sobresalientes de cualquier cultura, teniendo en cuenta además que las personas del común ven como algo normal el uso de manta por parte de un indígena y que por tal motivo no deben hacer luchas por alcanzar el respeto o la aceptación de esta costumbre, es por esto que ya algunos mayores, docentes y algunos pobladores están usando su manta lo cual indica un buen inicio y un ejemplo para el resto del pueblo para que se animen a recuperar lo que por derecho les pertenece sin pena, sino que por el contrario se haga con orgullo de mostrar lo que se es en realidad.

El modelo educativo de la etnoeducación dentro del pueblo Kankuamo está orientado a impartir el conocimiento propio de una escuela occidental, como son llamadas por los indígenas las escuelas que no pertenecen a ningún grupo indígena, $\mathrm{y}$ que cada docente sin importar la asignatura que enseñe, éstas se orienten fortalecimiento de la cultura. Si bien es cierto que los docentes saben de la importancia de la formación académica dentro de un proceso de formación eficiente para poder acceder a una educación superior, éstos también son garantes de que la formación que ellos impartan también incluya el fortalecimiento y rescate de la cultura propia, ya que si un estudiante es bien formado académicamente y posee 
ese amor y sentido de pertenencia hacia su cultura en la medida que estos tengan la oportunidad de formarse como profesional no solamente va a ser competitivo, sino que además este luchará por el desarrollo y la prosperidad de su pueblo.

\section{Identidad Cultural}

El proceso de la identidad cultural desarrollado dentro de la Institución es de vital importancia dentro de la formación del individuo, pues es en este contexto donde se aprende que, en cualquier ciudad, país, municipio, departamento, pueblo y hasta barrio, la identidad cultural es distinta y que del mismo modo como se valora la cultura propia se debe valorar y respetar la de los demás, y que esto es fundamental para poder convivir dentro de cualquier contexto con otras personas, porque una persona que no tiene claras estas pautas para la sana convivencia y respeto hacia los demás será a futuro una persona agresiva, intolerante, que considera que solo él vale por lo que es y de donde es y se convertiría en un problema más para la sociedad actual que de lo único que está ávida en estos momentos es de personas con valores y principios.

En relación al nuevo modelo educativo, algunos docentes lo explican basados en primera instancia en el interrogante: "la educación moldea la sociedad, o si por el contrario la sociedad moldea la educación”. El pueblo indígena Kankuamo decidió contar con la colaboración del pueblo en el desarrollo de un nuevo proyecto para la educación que constituyera no solo un modelo nuevo de educación, sino que además incluyera la formación a los estudiantes en la cultura propia, y adicional a esto se elaborara con la participación de muchas personas pertenecientes a esta cultura donde se compartieron, expusieron y debatieron muchas ideas hasta llegar a lo que hoy se conoce como Proyecto Educativo Kankuamo (PEK), proyecto que para las instituciones y docentes ha dado hasta hoy buenos resultados sin desconocer que este proceso será arduo y constante para alcanzar los objetivos trazados.

Dentro del PEK hay cinco proyectos tendientes al fortalecimiento de la cultura propia, y una de ellas es el proyecto de identidad, en el que los estudiantes ven asignaturas orientadas a la enseñanza de costumbres propias de su cultura. También existe una asignatura llamada autoprotección, que imparte conocimiento acerca de los principios y los valores, este modelo educativo es sin lugar a duda interesante e importante en la formación de esta cultura. Si se comparara este modelo educativo con relación a un modelo de educación distinta a esta, se haría clara la brecha entre ambas si se observan paralelamente; el PEK se da en una población rural y por tal razón algunos aspectos que ayudan al desarrollo de la formación en lo académico son más limitados el acceso a internet, a bibliotecas públicas, a ir a una librería, a la compra de algunos materiales, la situación económica este aspecto lo tiene en contra el estudiante Kankuamo, la clasificación de estudiantes citadinos por estratos y que en ese mismo nivel se imparta el conocimiento es algo desfavorable para estos. En el pueblo todos van a la misma escuela, a los estudiantes Kankuamos se les enseña de manera oral, escrita y práctica todo lo relacionado con su cultura, danzas, juegos, costumbres, palabras, creencias. La escuela citadina imparte religión no católica, no cristiana, porque la diversidad de creencia prohíbe que sea una religión específica, a menos que la escuela tenga en su razón social que es católica o cristiana, obviamente quienes se inscriban en una de estas dos tienen este tipo de creencias.

La historia de Colombia en general que todo estudiante conoce, ética y valores, los generales para la sana convivencia en la sociedad, son una ventaja para los estudiantes Kankuamos; conocer la cultura de la que también hacen parte, porque viven dentro de un país, pero adicional a ello conocen muy de cerca y tienen la oportunidad de vivir y aprender de su propia cultura dentro de una institución lo cual constituye un valor agregado en la formación del estudiante.

El proceso de reconstrucción cultural, por llamarlo de algún modo, es un proceso arduo pues se trata de recoger partes fragmentadas de una cultura que es lo único que existe hoy, aunque hace varias décadas era una cultura solida, y que el tiempo y la influencia de diversos factores moldearon de tal modo que solo dejaron vestigios de la que fuera una de las culturas más 
arraigadas y que por culpa de tanta influencia y sin que sus mismos habitantes se dieran cuenta se fue perdiendo en el tiempo y por la indiferencia de quienes pertenecen a esta. Pero a pesar de todo esto algunas personas han despertado y han visto con preocupación la necesidad de hacer algo para que su cultura no desaparezca, sino que por el contrario con lo poco que queda vivo de ella vuelva a florecer, que sea como como esa llama que ardió vivazmente durante mucho tiempo pero que a medida que la leña se iba acabando se fue apagando hasta quedar una llama tan pequeña que es casi imperceptible, llama que hoy el pueblo Kankuamo mediante el PEK, pretende avivar para que todos puedan verla, que no pase desapercibida ante la vista de nadie, que se fortalezca y que sea un modelo a seguir para muchas culturas que se hallan en esta situación.

Aunque para algunos es indiferente el querer renacer, para otros sí se les han vuelto atractivos todos los beneficios otorgados por la Constitución a los pueblos minoritarios y sobre todo a los indígenas y aquellos que antes eran apáticos a que los llamaran indígenas ahora "sí quieren pero no", o sea que no quieren que los vean como indígenas, pero sí quieren recibir los beneficios porque al fin y al cabo sí son indígenas, es el caso que para los estudios universitarios de sus hijos piden a las autoridades indígenas las certificaciones de su descendencia para ser sujetos de los beneficios que este hecho les otorga.

Entre los docentes de los colegios, donde se imparte la etnoeducación dentro del Cabildo Kankuamo, existe unidad de criterios para llevar adelante el proceso de transformación educativa que necesita toda esta población y coadyuvar con sus conocimientos para que todo este proceso dé los frutos deseados, ellos le apuestan al proyecto etnoeducativo y dicen que después que se tenga todo el apoyo estatal, este proceso sale adelante, y que no comparten el temor que tienen algunos padres de familia con respecto a la educación que se les está impartiendo a sus hijos en estas instituciones educativas ya que además de impartirles todo lo concerniente al PEI (Proyecto Educativo Institucional), también se les imparte lo concerniente al PEK (Proyecto Educativo Kankuamo), lo que garantiza que el estudiantado debe salir mejor fortalecido con esta clase de educación, ya que además de conocer todo lo referente a las materias del currículo normativo institucional, se les fortalece con mayor intensidad horaria para abordar lo referente al PEK, como son las costumbres, la religión, la lengua, las políticas, su cosmovisión, el amor a la tierra y el respeto a sus ancestros, también lo referente a la cultura, sus danzas, sus creencias, sus medicamentos, sus manufacturas, sus cultivos, sus comidas, sus viviendas y las sanas costumbres.

\section{CONCLUSIÓN}

Para nosotros, la etnoeducación es la más acertada para el pueblo indígena Kankuamo, en este caso porque abarca todo lo concerniente a lo institucional y a lo étnico y en consonancia con esto el estudiantado debe salir más fortalecido en estas competencias y por supuesto tener mejores logros a nivel comparativo y competitivo con los estudiantes de otras instituciones educativas y así poder acceder a las mejores universidades nacionales e internacionales aprovechando todo el apoyo que están prestando gobiernos extranjeros a las minorías étnicas, lo que hay es que trabajar unificados y fortalecer lo existente y alejar odios y rencillas de carácter personal y políticos en aras del futuro de los niños y niñas del Pueblo Kankuamo.

Las diversas expresiones de la realidad sociocultural del pueblo indígena Kankuamo se pueden revitalizar conservando las costumbres y tradiciones desde los procesos etnoeducativos que el PEK puede abordar, integrando a toda la comunidad educativa alrededor de la música tradicional -La gaita y el chicote $^{1}$-, la recuperación de los lugares de pagamento ${ }^{2}$, la reflexión de la medicina tradicional, la celebración del Corpus Christi en el mes de junio.

1. Son instrumentos de vientos construidos con carrizo. Según algunos autores, el chicote se interpretaba durante las ceremonias religiosas.

2. En la Sierra, el pagamento es un acto de alimentar a los ancestros, de pagarles; designa igualmente los lugares donde se realizan estos actos. 
En este sentido, pero desde una mirada más crítica, la etnoeducación como motor de desarrollo de la sociedad (no solo de la kankuama) debe posibilitar la adopción y afianzamiento en cada persona y grupo de una visión y pensamiento enfocado a la "convivencia pacífica con identidades culturales e ideológicas diversas, porque con individuos aislados, sin referentes claros, se hace difícil una construcción social sólida" Bartolomé (2002).

Por ello, la etnoeducación y en particular quienes la lideran en las comunidades, deben ser orientados sobre la educación intercultural como base de esta, a través de procesos formativos.

\section{REFERENCIAS BIBLIOGRÁFICAS}

Arango, A.; Aristizabal, S. (1991). Proyecto etnoeducativo en la cultura Embera-Chamí. Medellín, Colombia. Colciencias-Universidad de Antioquia.

Bartolomé, M.; Cabrera, F.; Del Campo, J.; Espín, J.V.; Marín, M.A.; Rodríguez, M. (2002). Identidad y ciudadanía. Un reto a la educación intercultural. Madrid, España. Narcea.

Ferrando, M.; Ibáñez, J.; Alvira, F. (1994). El análisis de la realidad social, métodos y técnicas de investigación. Alianza Universidad textos.

Giddens, A. (1984). The constitution of society. University of California.

Krueguer, R. (1991). El Grupo de Discusión, guía práctica para la investigación aplicada. Madrid, España. Pirámide. 